\title{
Research on one-direction receiving method of guided waves based on magnetostrictive effect
}

\author{
Jiang Xu \\ School of Mechanical Science and Engineering \\ Huazhong University of Science and Technology \\ Wuhan, P.R. China \\ e-mail: jiangxu@hust.edu.cn
}

\author{
Xinjun Wu \\ School of Mechanical Science and Engineering \\ Huazhong University of Science and Technology \\ Wuhan, P.R. China \\ e-mail: xinjunwu@hust.edu.cn
}

\author{
Yufeng Zhang \\ Jiangsu Transportation Institute \\ Nanjing, P.R. China \\ e-mail: kolya@jsti.com
}

\author{
Zhen Sun \\ Jiangsu Transportation Institute \\ Nanjing, P.R. China \\ e-mail: sz21@jsti.com
}

\begin{abstract}
The guided wave with the bidirectional propagation leads to complex signal which would be difficult to identify the end echo and the flaw echo. To simplify the guided wave signals based on the magnetostrictive effect, one-direction receiving method is investigated. The receiving guided wave method in one direction is obtained from the beam superposition by considering the position of the receiving coils and the direction of the wave propagation. The process of the signal is given in detail. The method is verified by the experiments. Two transmitters and two receivers are applied at the same time which could increase the signal-to-noise ratio by about four times than one transmitter and one receiver.
\end{abstract}

Keywords- one-direction receiving method; guided wave; magnetostrictive effect; ultrasonic; nondestructive testing

\section{INTRODUCTION}

The guided wave inspection technology has widely accepted as a quickly effective nondestructive testing method [1-5]. Guided waves can travel long distance from the excited position with low attenuation. Several technologies are applied to generate guided waves, such as piezoelectric, laser, Lorentz force and magnetostrictive technology. The non-contact magnetostrictive technology can generate guided waves with a large lift-off distance, especially to coated pipes and stay cables [6]. Due to the multi-mode and disperse characteristic, the testing signals are very complex. Moreover if the excitation position is in the middle of pipes, waves traveling two directions are generated and received. The signals will be much more complicated. Also, many researchers focus on how to obtain the simple signals, such as the single mode, the nondisperse mode, the unidirectional propagated waves [7].

The unidirectional inspection technology simplifies the signals by reducing the source of waves and counteracting special direction waves. The method could not only cancel one direction waves but also enhance the other direction waves. Bottger et al. employ two identical excitation coils to obtain the unidirectional propagated waves by using an electromagnetic acoustic transducer system [8]. The center distance of two coils is a quarter-wavelength. The excited signal applied to the two coils is time shifted by a quarter period of the signal. Tang et al. employ two identical excitation coils to obtain the unidirectional propagated waves by using a magnetostrictive guided wave system [9]. Alleyne et al. applied two dry piezoelectric transducer rings to obtain the unidirectional propagated waves [10]. A simple theoretical analysis of beam forming was given. They concluded that the amplitude the unidirectional propagating wave is double obtained with a single ring. However, there is little information available in literature about the unidirectional receiving guided waves. It is hoped that the technology could be understood and mastered easily and clearly for other researchers with our study. The aim of this paper is to provide the detail of implement the unidirectional receiving signal based on magnetostrictive effect.

\section{THEORY}

Fig .1 shows the schematic diagram of receiving guided waves base on magnetostrictive effect in pipes or cables. Consider two receivers, $R_{1}$ and $R_{2}$, position at $x=0$ and $x=L_{1}$ respectively. The length of the receivers is supposed close to zero. Here we consider two waves from different direction. The $A$ wave is from left side and the start time is $t_{l}$ when the wave passes $R_{l}$. The $B$ wave is from right side and the start time is $t_{2}$ when the wave passes $R_{2}$.

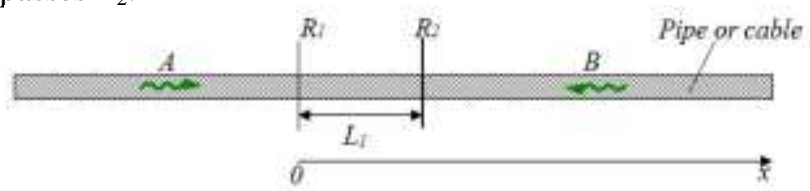

Figure 1. Schematic diagram of receiving guided waves

The signal receives by $R_{1}$ and $R_{2}$ induced by $A$ are shown as

$$
V_{A 1}=\sin \omega t \quad t_{1} \leq t \leq t_{1}+n T
$$




$$
V_{A 2}=\left(1-\alpha L_{1}\right) \sin \omega t \quad t_{1}+L_{1} / v_{1} \leq t \leq t_{1}+L_{1} / v_{1}+n T
$$

The signal receives by $R_{1}$ and $R_{2}$ induced by $B$ are shown as

$$
\begin{gathered}
V_{B 1}=\left(1-\alpha L_{1}\right) \sin \omega t \quad t_{2}+L_{1} / v_{1} \leq t \leq t_{2}+L_{1} / v_{1}+n T \\
V_{B 2}=\sin \omega t \quad t_{2} \leq t \leq t_{2}+n T
\end{gathered}
$$

If the data of $R_{1}$ shifts $+L_{1} / v_{1}$ of time, we can obtain that

$V_{A 1}^{\prime}=\sin \omega t \quad t_{1}+L_{1} / v_{1} \leq t \leq t_{1}+L_{1} / v_{1}+n T$

$V_{B 1}^{\prime}=\left(1-\alpha L_{1}\right) \sin \omega t \quad t_{2}+2 L_{1} / v_{1} \leq t \leq t_{2}+2 L_{1} / v_{1}+n T$

If the distance of two receivers $L_{l}$ is very short, the $\alpha L_{2}$ is close to zero. The data of $R_{1}$ ' subtracts the data of $R_{2}$ is

$$
\begin{aligned}
& V_{A}=0 \quad t_{1}+L_{1} / v_{1} \leq t \leq t_{1}+L_{1} / v_{1}+n T \\
& V_{B}=V_{B 1}^{\prime}-V_{B 2} \quad t_{2} \leq t \leq t_{2}+2 L_{1} / v_{1}+n T
\end{aligned}
$$

As shown in (7) and (8), the method can realize the right side unidirectional receiving guided waves. We can obtain the left side unidirectional receiving guided waves in the same method. If the $L_{l}$ is very large, the $\alpha L_{l}$ doesn't close to zero. The other direction signal could not be canceled. Therefore the $L_{l}$ is the smaller the better. The best distance of the receivers is a quarter-wavelength. The process of signal superposition is shown in Fig .2. From Fig .2, the amplitude of the superposition wave is double that received with a single receiver except the first half period and the last half period.

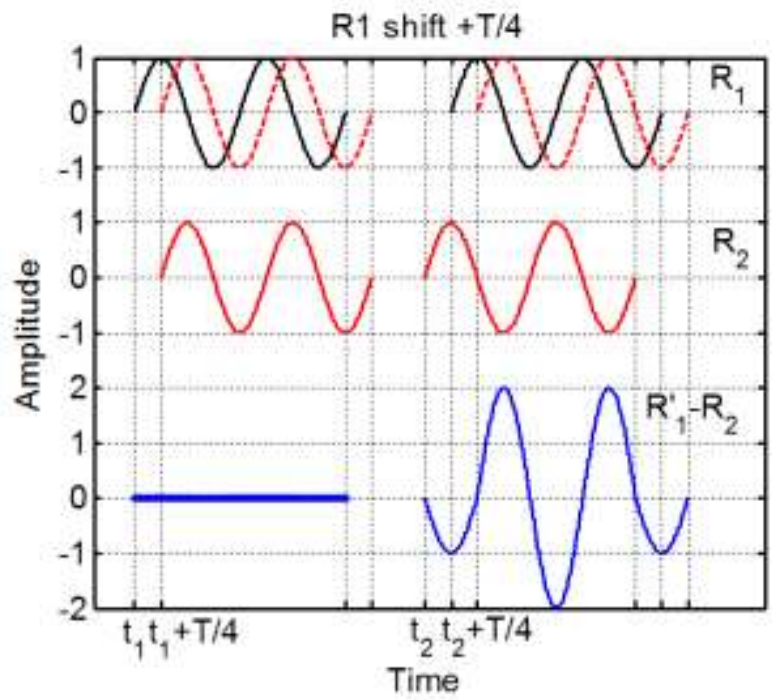

(a) Cancel the signal from left direction

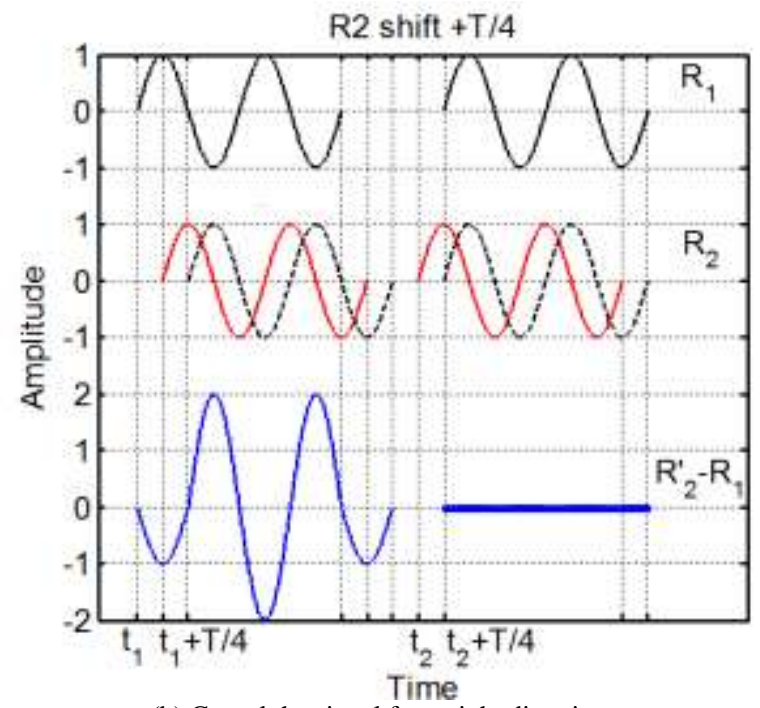

(b) Cancel the signal from right direction

Figure 2. Process of receiving signal superposition

\section{EXPERIMENT VERIFICATION}

The inspection system includes dual-channel signal generator and dual-channel signal acquisition. The generators with precise delay control could generate specified frequency and cycle tone burst sinusoidal signal. The transducers are for generating longitudinal mode guided waves. A sample of approximately $3 \mathrm{~m}$ long, $15.24 \mathrm{~mm}$ diameter prestressed steel strand was used in the experiment. The exciting frequency is $87 \mathrm{kHz}$. The group speed of these frequencies is about $5200 \mathrm{~m} / \mathrm{s}$. The wavelength of wave is approximately $60 \mathrm{~mm}$. The coil for one transmitter or receiver is $15 \mathrm{~mm}$ width flat cable. The number of turns is ten.

Experiment 1: one transmitter and one receiver work, the data and the wave propagation path are shown in Fig .3. The first signal is initial pulse applied to the transmitting coil which is electrically leaked to the receiving coil from the air at the speed of light. The bidirectional waves were generated in the pipe. Firstly, the right propagation wave was induced which was shown as "first passing signal". Secondly, the left propagation wave reflected from left end was induced which was shown as "first left end echo". Thirdly, the right propagation wave reflected from right end which was shown as "first right end echo". Fourthly, the left propagation wave reflected from right end was induced which was shown as "second right end echo". The waves would propagate in the pipes until decaying to zero. If there are some flaws or weld in the pipe, the signals would be very complex. The bidirectional waves would reflect from at location where there is a change of crosssection or a change in local stiffness of the pipe. 

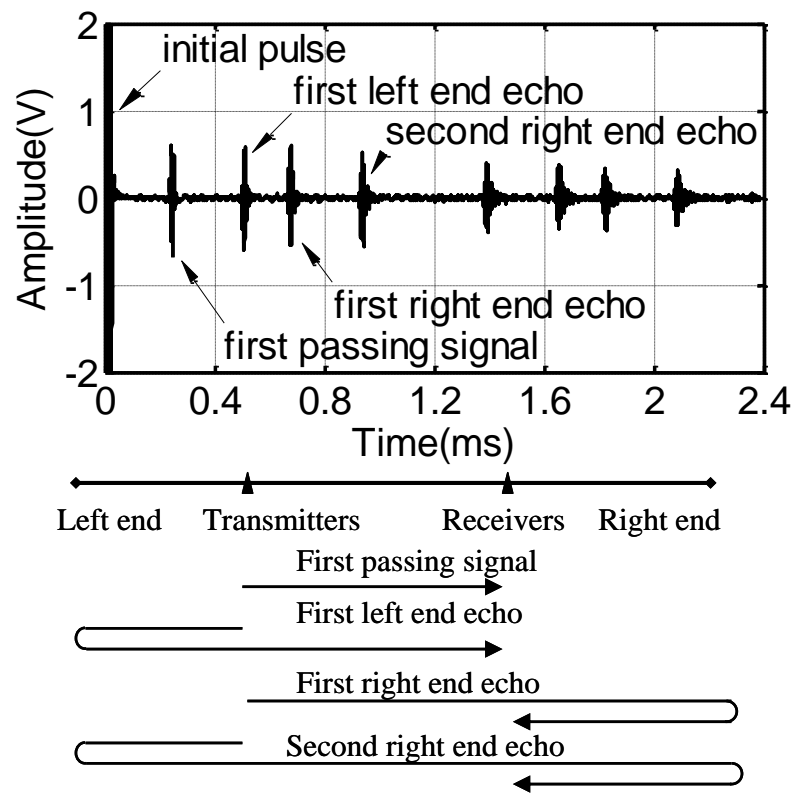

Figure 3. Data and wave propagation path of one transmitter and one receiver

Experiment 2: two transmitters and two receivers work. The input signal of the left transmitter is a pulse in $87 \mathrm{kHz}$ with two sinusoidal cycles. The input signal of the right transmitter is the same as left transmitter but with a delay time of $2.875 \mu \mathrm{s}$. The received data of two receivers are shown in Fig .4. The left propagated wave was generated and the data include "first left end echo" and "second right end echo" only. Then the transmitted signals of two transmitters were swap. The received data are shown in Fig .5. The right propagated wave was generated and the data include "first passing signal" and "first right end echo" only.

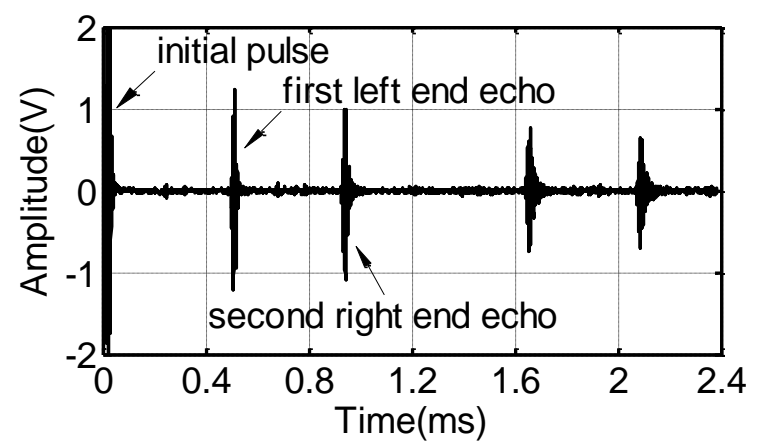

(a) Induced by left receiver

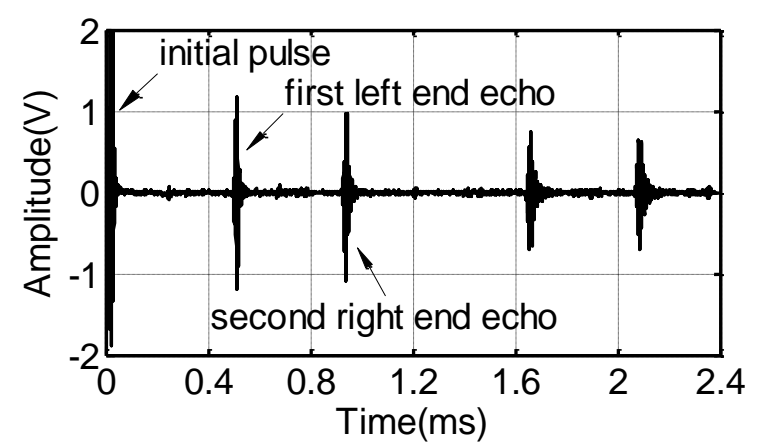

(b) Induced by right receiver

Figure 4. Data of the direction control to left by two transmitters

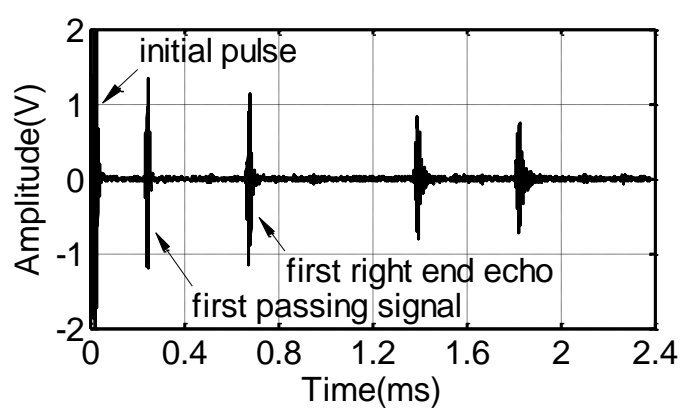

(a) Induced by left receiver

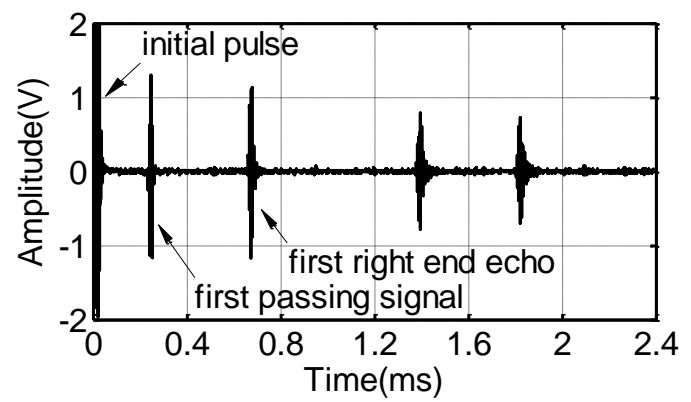

(b) Induced by right receiver

Figure 5. Data of the direction control to right by two transmitters

The method shown in Fig .2 is applied. The shift time of Fig .4 (b) is $+2.875 \mu$ s. Then the shifted data subtracts the data of Fig .4 (a) and the result is shown in Fig .6 (a) We obtain the data which only includes the signal from the left side of the receivers. The shift time of Fig .4 (b) is $-2.875 \mu$ s. Then the shifted data subtracts the data of Fig .4 (a) and the result is shown in Fig .6 (b). We obtain the data which only includes the signal from the right side of the receivers. Using the same method, the unidirectional receiving data are shown in Fig .7 from data of Fig .5.

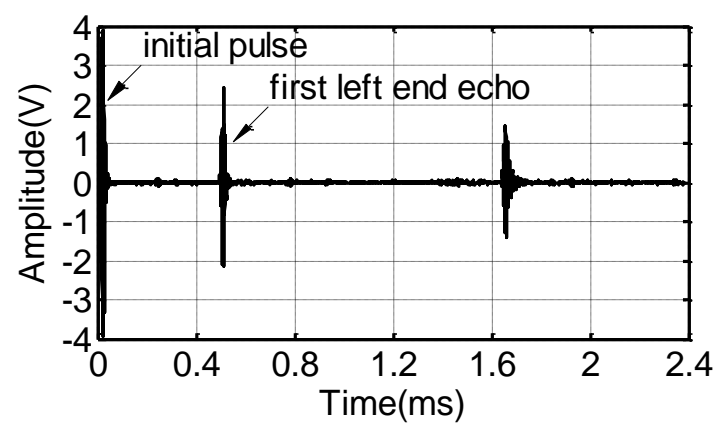

(a) From left side of receivers

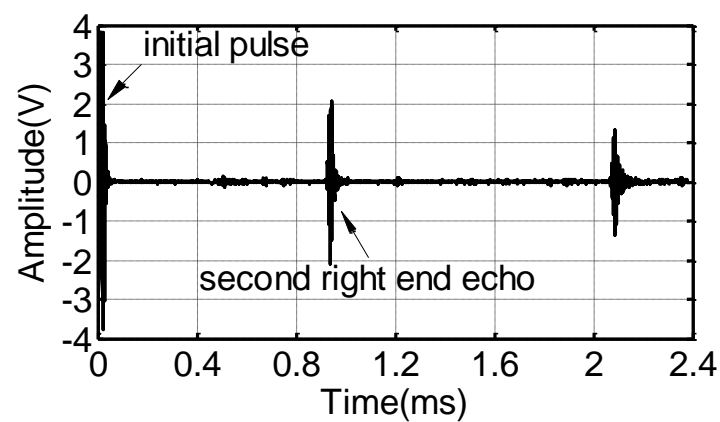

(b) From right side of receivers

Figure 6. Data of the left direction control to processed by time delay method 


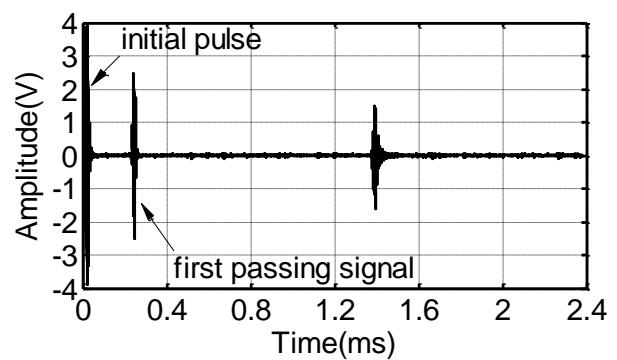

(a) From left side of receivers

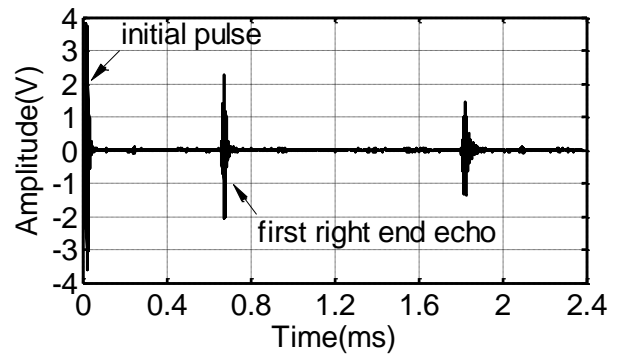

(b) From right side of receivers

Figure 7. Data of the right direction control to processed by time delay method

\section{DISCUSSIONS}

The method can realize the unidirectional receiving guided waves. As shown in Fig .7, when the only two transmitters are used, other direction waves cannot be canceled completely. The reason is that the effect of the dispersion. The magnetostritive guided wave technology is a wide band testing method. It is hard to completely avoid the dispersion when the L mode guided waves are generated. Although the little disperse frequency band is chosen, the tiny disperse phenomenon cannot be avoided totally.

Comparing the data of "the first left end echo" between bidirectional and unidirectional generated guided waves. The max peak-peak value of unidirectional signal is about twice as the bidirectional signal. The results agree with the above theory in general. The Initial segment waveforms of "the first left end echo" overlap each other. But the difference of waveforms increases gradually and then decreases gradually, not always twice. Lastly the waveforms overlap again. The amplitude of signal is increase more than twice. However the frequency of signals is changed, from high frequency to low frequency. The magnetostrictive guided wave technology is a broadband detection method. There is a frequency band of the signals, especially the low frequency under the induced frequency. Therefore the changing of signals does not meet the theory. If the cycle of the excited signal is large (up to 10) and the tone burst is modulated by a Hanning or Kaiser window, the influence of other frequency band would be weakened. Their results will agree well with the theory. As the use of the window function, the phenomenon of the start and the end signals would be hardly to discover because the start and the end of signals will be attenuated. Consequently the phenomenon was not mentioned in the Alleyne's paper.

Compare Fig .4-Fig .7, the peak-peak value of the echoes is increased when two transmitters and two receivers are applied. Further the amplitude of noise which is about $0.06 \mathrm{v}$ does not change. Table 1 shows the signal-to-noise ratio (SNR) changing under different situation. The SNR of two transmitters and one receiver is improved about twice than one transmitter. And the SNR of two transmitters and two receivers is improved about fourfold than one transmitter which is consistent with the theory.

Table 1 SNR under different situation

\begin{tabular}{|c|c|c|c|c|c|}
\hline $\begin{array}{l}\text { direction of } \\
\text { propagation }\end{array}$ & $\begin{array}{l}\text { receiving } \\
\text { mode }\end{array}$ & $\begin{array}{c}\text { first } \\
\text { passing } \\
\text { signal }\end{array}$ & $\begin{array}{l}\text { first } \\
\text { left } \\
\text { end } \\
\text { echo }\end{array}$ & $\begin{array}{c}\text { first } \\
\text { right } \\
\text { end } \\
\text { echo }\end{array}$ & $\begin{array}{l}\text { second } \\
\text { right } \\
\text { end } \\
\text { echo }\end{array}$ \\
\hline bi-direction & $\begin{array}{l}\text { single } \\
\text { receiver }\end{array}$ & 21.4 & 19.8 & 18.9 & 17.9 \\
\hline to left & $\begin{array}{l}\text { single } \\
\text { receiver }\end{array}$ & - & 39.5 & - & 34.7 \\
\hline to left & $\begin{array}{l}\text { double } \\
\text { receivers }\end{array}$ & - & 75.7 & - & 68.9 \\
\hline to right & $\begin{array}{l}\text { single } \\
\text { receiver }\end{array}$ & 42.4 & - & 38.2 & - \\
\hline to right & $\begin{array}{l}\text { double } \\
\text { receivers }\end{array}$ & 82.9 & - & 72.0 & - \\
\hline
\end{tabular}

\section{CONCLUSIONS}

The method for one-direction receiving guided waves based on magnetostrictive effect was investigated in this study. To obtain the unidirectional receiving signal, two receivers need to be applied with a special spacing and a difference algorithm with an offset time. Moreover an inspection system has also been developed which gives unidirectional propagation of longitudinal and torsional modes base on magnetostrictive effect. The theoretical analysis and the experiment results indicate that the amplitude of the unidirectional receiving wave is double obtained with one receiver. Two transmitters and two receivers are applied at the same time which could increase the SNR by about four times than one transmitter and one receiver. This study simplifies the received signals. Further the results imply that the method could increase the detection accuracy.

\section{ACKNOWLEDGMENT}

This work was supported by the National Natural Science Foundation of China (Grant No. 51205148), the Special Funds of Non-profit Industry Quality Supervision for Research (Grant No. 201310157) and the Special Fund for Basic Scientific Research of Central Colleges (Grant No. 2014TS039).

\section{REFERENCES}

[1] J.L. Rose, Ultrasonic Guided Waves in Solid Media. Cambridge University Press, New York, 2014.

[2] Z. Liu, J. Zhao, and B. Wu, "Configuration optimization of magnetostrictive transducers for longitudinal guided wave inspection in seven-wire steel strands," NDT and E International, vol. 43, July, 2010,pp. 484-492, doi: 10.1016/j.ndteint.2010.05.003.

[3] X. Wang, P.W. Tse and C.K. Mechefske, "Experimental investigation of reflection in guided wave-based inspection for the characterization of pipeline defects", NDT and E International, vol. 43, June, 2010,pp. 365-374, doi: 10.1016/j.ndteint.2010.01.002.

[4] H. W. Kim, J. K. Lee, and Y. Y. Kim, "Circumferential phased array of shear-horizontal wave magnetostrictive patch transducers 
for pipe inspection," Ultrasonics, vol. 53, Feb. 2013, pp. 423-431, doi: 10.1016/j.ultras.2012.07.010.

[5] J. Hua, J. Mu, and J.L. Rose, "Guided wave propagation and focusing in multi-layer pipe with viscoelastic coating and infinite soil media”, Materials Evaluation, vol. 71, May, 2013, pp. 369-377.

[6] J. Xu, X. Wu, C. Cheng, and A. Ben, "A Magnetic Flux Leakage and Magnetostrictive Guided Wave Hybrid Transducer for Detecting Bridge Cables,” Sensors, vol. 12, Jan. 2012, pp. 518-533, doi: 10.3390/s120100518.

[7] D. N. Alleyne, T. Vogt, and P. Cawley, "The choice of torsional or longitudinal excitation in guided wave pipe inspection," Insight Non-Destructive Testing and Condition Monitoring, vol. 51, Jul. 2009, pp. 373-377, doi:10.1784/insi.2009.51.7.373.
[8] W. Böttger, H. Schneider, and W. Weingarten, "Prototype EMAT system for tube inspection with guided ultrasonic waves," Nuclear Engineering and Design, vol. 102, Jul. 1987, pp. 369-376, doi:10.1016/0029-5493(87)90183-x.

[9] H. Tang, J. Xu and X.J. Wu, "Research on Unidirectional Transmitting Method of Magnetostrictive Guided Wave," Proc. IEEE, 3rd International Conference on Computational Intelligence and Industrial Application, IEEE Press, Aug. 2010, pp. 125-128.

[10] D.N. Alleyne and P. Cawley, "Long Range Propagation of Lamb Waves in Chemical Plant Pipework", Materials Evaluation, vol. 45, April, 1997, pp. 504-508. 